\title{
Diagnostic performance of non-invasive fractional flow reserve derived from coronary computed tomography angiography: current perspectives
}

This article was published in the following Dove Press journal:

Journal of Vascular Diagnostics and Interventions

29 April 2016

Number of times this article has been viewed

\author{
Mohamed Ahmed \\ Nikolaos Kakouros \\ Department of Medicine, Division \\ of Cardiology, University of \\ Massachusetts Medical School, \\ Worcester, MA, USA
}

Correspondence: Nikolaos Kakouros Division of Cardiovascular Medicine, University of Massachusetts Medical School, 55 Lake Avenue North, Worcester, MA 0I655, USA

Tel + I 7744425043

Fax + I 774 44I 6303

Email Nikolaos.Kakouros@umassmed.edu
Abstract: Coronary computed tomography angiography (CTA) has been increasingly used to detect coronary artery disease. The diagnostic performance of coronary CTA is well established with a high sensitivity and negative predictive value. Nevertheless, the diagnostic value of coronary CTA is offset by a high false positive rate, partly due to the technique lacking physiological lesion assessment when performed in the conventional way. This has raised concerns regarding unnecessary invasive coronary angiography referrals and inappropriate revascularization procedures. Recent advances in computational fluid dynamics and image-based modeling have enabled the calculation of coronary artery blood flow and pressure under various modeled physiologic conditions from coronary CTA without the need for hyperemia-inducing medications, modification of acquisition protocol or further radiation. Coronary flow and pressure can be derived both at rest and during simulated maximum hyperemia allowing for the calculation of fractional flow reserve from coronary $\mathrm{CTA}\left(\mathrm{FFR}_{\mathrm{CTA}}\right)$ across stenotic lesions in a fashion similar to invasive FFR. This novel non-invasive technology offers concurrent anatomical and functional assessment of major epicardial coronary arteries. The diagnostic performance of FFR $_{\text {CTA }}$ has been tested in three major trials where it resulted in accurate identification of ischemia-related lesions. Similar to an invasive FFR-guided management strategy, the use of FFR $_{\mathrm{CTA}}$ has been shown to improve patients' outcomes and reduce health care costs. FFR ${ }_{\text {CTA }}$ is emerging as an attractive alternative to invasive FFR. There are, however, several challenges that need to be overcome before $\mathrm{FFR}_{\mathrm{CTA}}$ can be incorporated into routine clinical practice.

Keywords: computational Fluid Dynamics, CFD, coronary CTA, FFR, coronary artery disease, CAD

\section{Introduction}

Coronary artery disease (CAD) is a worldwide leading cause of mortality and morbidity. ${ }^{1}$ It is estimated that more than half of cardiovascular deaths are classified as sudden cardiac death, usually as a result of acute myocardial infarction in patients with underlying atherosclerotic CAD. ${ }^{2}$ Invasive coronary angiography (ICA) utilizing intracoronary injection of radiopaque contrast to obtain radiographic luminograms of the coronary vessels is currently the "gold standard" technique used in clinical practice to establish the coronary anatomy, and remains the cornerstone for the decision making process of choosing medical therapy versus percutaneous or surgical revascularization. Visual assessment of coronary luminal stenoses assumes a constant relationship between the degree of stenosis and ischemia as explained by Gould et al, whereby the flow to the myocardium is gradually compromised as the luminal diameter progressively narrows. ${ }^{3}$ Nonetheless, this relationship has been proven to be more complex 
and visual assessment of coronary luminal stenosis has been shown to poorly correlate with ischemia, particularly for lesions of moderate severity ( $40 \%-70 \%$ stenosis). ${ }^{4,5}$

Fractional flow reserve (FFR) is based on the relationship between coronary artery pressure and blood flow. ${ }^{6}$ Although this relationship is fairly variable at rest, during maximal hyperemia there is a linear relationship between coronary pressure and flow because myocardial resistance is minimal and therefore constant. ${ }^{7}$ FFR is defined as the ratio of maximal achievable blood flow in a coronary artery in the presence of a stenosis to the hypothetical maximal achievable blood flow in that same epicardial artery in the absence of the stenosis. ${ }^{8}$ It is calculated during invasive cardiac catheterization by measuring pressure beyond the stenotic lesion. This is most commonly achieved using a guide wire with a pressure-sensing transducer at its distal end, although alternative methods such as optical interferometric sensors can also be employed. After induction of maximal hyperemia using an epicardial as well as a microvascular vasodilating agent (often nitroglycerine and adenosine), the pressure gradient across the lesion is recorded and FFR is calculated as the minimal stable ratio of the mean distal coronary pressure to the mean aortic pressure. ${ }^{9,10}$ Pressure reduction of more than $25 \%$ at the distal vessel $(\mathrm{FFR}<0.75)$ correlates well with objective myocardial ischemia, whereas relative pressure reductions in the distal vessel of less than $20 \%$ (FFR $>0.80)$ rarely do. ${ }^{11}$

There is a wealth of data demonstrating the superiority of FFR-guided revascularization and improved event-free survival when compared to standard angiography-guided revascularization. ${ }^{12-17}$ In a 5-year follow-up of the DEFER study, percutaneous coronary intervention (PCI) of a functionally non-significant stenosis, as indicated by an FFR $\geq 0.75$, was of no benefit from a prognostic or symptomatic point of view. ${ }^{13}$ In accordance with the results from the DEFER study, the FAME study identified a benefit for deferring PCI in patients with multivessel disease and functionally non-significant lesions (FFR $\geq 0.80$ ), with reduced rates of cardiac events at both 1 and 2 years. ${ }^{9,14}$ Similar conclusions were drawn in patients with stable CAD in the FAME2 trial. In this trial, FFR-guided therapy reduced the need for urgent revascularization in patients with stable CAD and functionally significant lesions. ${ }^{16}$

Therefore, the American College of Cardiology Foundation/ American Heart Association/Society for Cardiovascular Angiography and Interventions guideline for PCI has recommended the use of an FFR-guided strategy in patients with stable ischemic heart disease (class IIa recommendation, level of evidence: A). ${ }^{18}$ FFR-guided strategy carries an even stronger level of recommendation (class Ia recommendation, level of evidence: A) in the 2014 European guidelines on myocardial revascularization. ${ }^{19}$ Surprisingly, despite the unequivocally favorable data, invasive FFR is underutilized in clinical practice, as fewer than $10 \%$ of coronary revascularization procedures in the US utilize FFR, perhaps due to its invasive nature, risk of instrumentation, increased procedural time, and the use of pharmacologic agents. ${ }^{20,21}$ This underutilization underscores the need for novel non-invasive methods to evaluate both anatomical and functional aspects of coronary stenosis. Such non-invasive anatomic-physiologic testing prior to cardiac catheterization and intervention may improve patients' outcomes and reduce costs.

\section{Non-invasive imaging of CAD and coronary blood flow}

Coronary computed tomography angiography (CTA) has been increasingly used in clinical practice to detect CAD. It is estimated that 2.3 million coronary CTA examinations are performed annually in the US alone. ${ }^{22}$ The diagnostic accuracy of coronary CTA for identification of CAD has been demonstrated in several studies with a sensitivity and negative predictive value (NPV) approaching $99 \%$ in individuals without known CAD. ${ }^{23,24}$ Nevertheless, this high sensitivity is offset by a relatively low specificity and positive predictive value (PPV) (64\%-83\% and 48\%-86\% respectively). ${ }^{21,22}$ Despite the high resolution anatomical identification of obstructive CAD, coronary CTA, in its conventional application, cannot determine the hemodynamic significance of coronary lesions and frequently overestimates the degree of coronary stenosis. The results from two recently published trials, PROMISE and SCOT-HEART, provided evidence that the use of coronary CTA for chest pain evaluation enhanced the diagnostic accuracy and appropriately altered subsequent therapeutic plans when compared to other modalities of non-invasive functional testing. ${ }^{25,26}$ However, coronary CTA increased the rate of referral for ICA and revascularization by up to $50 \%$ with little understanding of the ischemia-producing lesions raising concerns regarding unnecessary ICA and possibly inappropriate revascularization procedures..$^{25,27,28}$ Moreover, a strategy of initial CTA was associated with higher radiation exposure than functional testing as suggested by the PROMISE trial (mean cumulative radiation exposure of $12.0 \mathrm{mSv}$ versus $10.1 \mathrm{mSv} ; P<0.001){ }^{23}$

Although non-invasive tests such as myocardial perfusion imaging with single-photon emission CT and positron emission tomography, as well as stress echocardiography 
provide functional assessment of CAD by identifying regions of suboptimal coronary flow and wall motion abnormalities, they do not directly visualize coronary lesions and carry a significant false positive and false negative rate. A homogeneous myocardial perfusion scan does not exclude the presence of $\mathrm{CAD}$, especially in the setting of multivessel CAD and balanced ischemia. ${ }^{29}$ Positron emission tomography quantification of the transmural extent of myocardial perfusion is still far from ideal, despite the improvement in spatial resolution.

The combination of anatomic and physiologic imaging from a single imaging test can overcome the limitations of predominantly anatomic (eg, conventional cardiac CTA) and functional stress modalities. Although the CT myocardial perfusion imaging hybrid serves this purpose, it is limited by significant radiation exposure and increased cost. ${ }^{30}$ Therefore, recent advances in imaging techniques and fluid dynamics have been applied in the calculation of FFR from coronary CTA $\left(\right.$ FFR $\left._{\mathrm{CTA}}\right)$.

\section{Techniques and scientific basis of FF $\boldsymbol{R}_{\text {СтA }}$ calculation}

Recent advances in computational fluid dynamics and imagebased modeling now enable the calculation of coronary artery blood flow and pressure under different physiologic conditions from coronary CTA, without the need for hyperemia induction, modification of acquisition protocol, or additional radiation exposure. Coronary flow and pressure can be derived both at rest and during simulated maximum hyperemia permitting the ability to calculate FFR $_{\text {CTA }}$ across stenotic lesions. The scientific basis for quantifying FFR ${ }_{\text {CTA }}$ has been described in detail by Taylor et al:31 it is based on the generation of physiologic models of coronary blood flow.

The principal element for the generation of a reliable and precise model of human coronary blood flow is accurate three-dimensional anatomic imaging of the aorta and the coronary circulation. In addition, computation of patient-specific coronary velocity and pressure under different physiologic conditions is feasible through mathematical methods to solve the governing equations of fluid dynamics utilizing basic concepts. ${ }^{32-34}$ The first concept is the calculation of total resting coronary blood flow relative to myocardial mass quantified on coronary CTA. The baseline coronary blood flow at rest is proportional to the oxygen demands of the subtended myocardium. The second concept is that the resistance of the microvasculature bed is inversely related to the size of the supplying vessel. ${ }^{35-37}$ The third concept is that the microvasculature has a predictable vasodilator response to adenosine. ${ }^{38}$
The governing equations, known as the "Navier-Stokes equations", are solved to compute coronary blood flow and pressure. Computation of millions of equations simultaneously, and repetition of the process for thousands of time intervals in a single cardiac cycle is required to compute a realistic patient-specific model of the coronary circulation. ${ }^{39}$ The numerical methods for solving fluid dynamics problems are known as computational fluid dynamics. ${ }^{31}$

In addition to solving the governing equations, it is necessary to define the domain of interest and boundary conditions that interface the modeled domain to the remainder of the circulation in order to construct a precise patient-specific blood flow model. The domain of interest is the segment where the blood is flowing (the lumen), and the relevant boundaries, extracted from cardiac CTA data, including the lateral surface, the inlet boundary (the aortic root), and the outlet boundaries of the ascending aorta and the coronary arteries. Boundary conditions are mathematical relationships between the variables of interest (eg, flow and pressure; see Figure 1). The final step in constructing a realistic model of coronary flow requires coupling of lumped parameter models of the heart, systemic circulation, and coronary circulation to a patient-specific model of the root of the aorta along with the coronary tree extracted from cardiac CTA data. Finally, maximum hyperemia is simulated by modeling the effect of adenosine on reducing the peripheral microvascular myocardial resistance downstream of the coronary artery stenosis to compute $\mathrm{FFR}_{\mathrm{CTA}}$. It has been shown that in coronary arteries with normal coronary flow reserve, total coronary resistance at maximum hyperemia drops to a quarter of the resting value with intravenous administration of $140 \mathrm{mg} / \mathrm{kg} / \mathrm{min}$ of adenosine. ${ }^{38} \mathrm{FFR}_{\mathrm{CTA}}$ is then computed as the ratio of the mean hyperemic pressure downstream of the coronary stenosis to the mean hyperemic pressure in the aorta.

\section{Diagnostic performance of FFR in correlation with invasive FFR}

Emerging data suggest that non-invasive calculation of lesion-specific FFR, as described in "Non-invasive imaging of CAD and coronary blood flow" section, is feasible using coronary CTA with high diagnostic performance. Using invasive FFR as a reference, the diagnostic accuracy of FFR $_{\text {CTA }}$ has been tested in three clinical trials. ${ }^{40-42}$ In the DISCOVER-FLOW study, 103 patients with known or suspected CAD underwent coronary CTA in four international centers. Obstructive CAD was defined as a stenosis $\geq 50 \%$ on coronary CTA and lesion-specific ischemia was defined as FFR or $\mathrm{FFR}_{\mathrm{CTA}} \leq 0.8$. FFR $_{\mathrm{CTA}}$ was computed in 159 major 


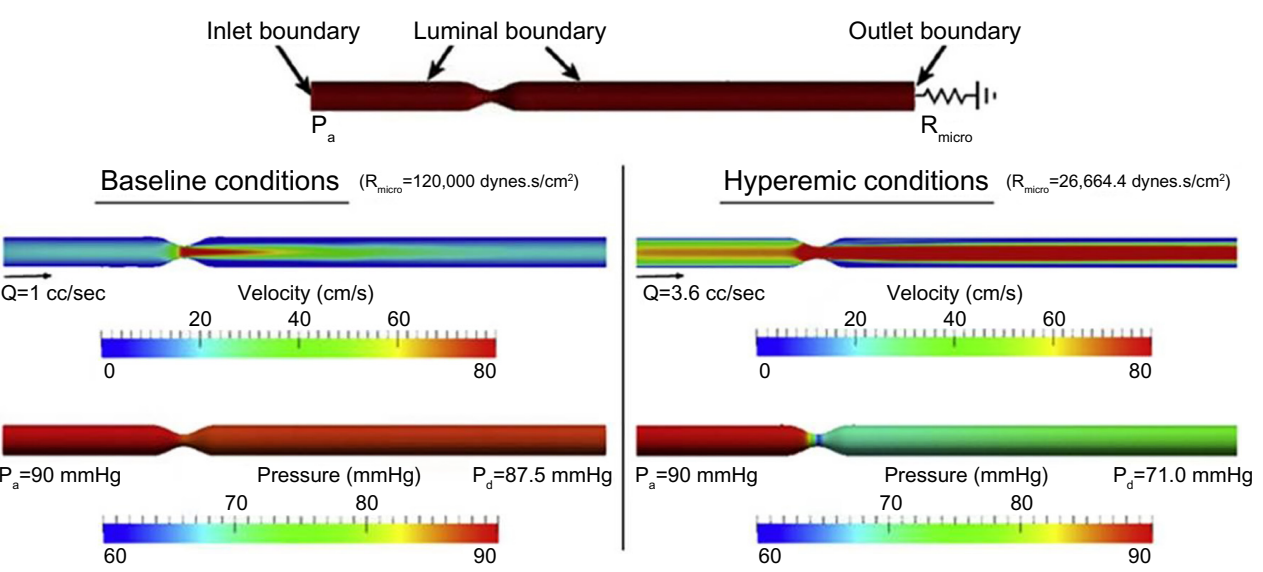

Figure I Mathematical model of blood flow through an idealized LAD stenosis with a reference diameter of $3.5 \mathrm{~mm}$ and $60 \%$ diameter reduction stenosis.

Notes: A constant pressure $\left(\mathrm{P}_{\mathrm{a}}=90 \mathrm{~mm} \mathrm{Hg}\right)$ is applied at the inlet boundary, and a constant resistance, $\mathrm{R}_{\text {micro }}$, is prescribed at the outlet boundary to simulate the downstream microcirculatory resistance. The velocity is set at zero along the luminal boundary. $R_{\text {micro }}$ is set at 120,000 dynes.s. $\mathrm{cm}^{-2}$ to model baseline conditions with a flow rate of $\mathrm{I} \mathrm{cc.s} \mathrm{s}^{-1}$ and is reduced by a factor of 4.5 to $26,664.4$ dynes.s. $\mathrm{cm}^{-2}$ to model hyperemic conditions. Hyperemic flow increases to $3.6 \mathrm{cc} . \mathrm{s}^{-1}$. The velocity on a slice along the vessel illustrates that a jet through the stenosis rapidly dissipates under baseline conditions but persists under hyperemic conditions. $\left(P_{d} / P_{a}=0.97\right.$ at baseline, $F F R=P_{d} / P_{a}=0.79$ at hyperemia). Figure reprinted from J Am Coll Cardiol, 22/6I, Taylor CA, Fonte TA, Min JK, Computational fluid dynamics applied to cardiac computed tomography for noninvasive quantification of fractional flow reserve: scientific basis, 2233-224I, Copyright 2013, with permission from Elsevier. ${ }^{31}$

Abbreviations: FFR, fractional flow reserve; LAD, left anterior descending.

coronary arteries and compared to FFR performed at the time of invasive angiography. FFR $_{\text {CTA }}$ correlated well with invasive FFR $(r=0.717, P<0.001)$ and was superior and additive to coronary CTA for the detection of ischemia-causing lesions. On a per-vessel basis, the sensitivity, specificity, PPV, and NPV were $87.9 \%, 82.2 \%, 73.9 \%, 92.2 \%$, respectively, for FFR $_{\text {CTA }}$ compared to $91.4 \%, 39.6 \%, 46.5 \%, 88.9 \%$, respectively, for coronary CTA stenosis. Evidently, the enhanced diagnostic accuracy of FFR $\mathrm{CTA}_{\mathrm{CA}}$ compared to coronary CTA ( $84 \%$ versus $59 \%$ ) is largely due to lower false positive rates observed with $\mathrm{FFR}_{\mathrm{CTA}} \cdot{ }^{40}$

The above findings were subsequently reproduced in a larger cohort, the DeFACTO study. ${ }^{41}$ Similar to the DISCOVER-FLOW study, 252 stable patients with known or suspected CAD in 17 international centers were evaluated with cardiac CTA, FFR ${ }_{\text {CTA }}$, and invasive FFR. Obstructive CAD was defined as a stenosis $\geq 50 \%$ and ischemia was defined as FFR or FFR CTA $\leq 0.8$. FFR CTA $_{\text {Cemonstrated supe- }}$ riority over coronary CTA in ischemia determination. The DeFACTO study's prespecified primary outcome goal was to demonstrate a per-patient diagnostic accuracy for FFR CTA of $>70 \%$ at the lower $95 \%$ confidence interval (CI) border of certainty. In per-patient analysis, sensitivity, specificity, PPV, and NPV were $90 \%, 54 \%, 67 \%$, and $84 \%$ with FFR compared to $84 \%, 42 \%, 61 \%$, and $72 \%$ with coronary CTA respectively. Although the study failed to meet its primary endpoint, FFR $_{\text {CTA }}$ demonstrated improved accuracy for the diagnosis of ischemia compared to coronary CTA alone; $73 \%(95 \%$ CI $67 \%-78 \%)$ for $\mathrm{FFR}_{\text {СTA }}$ versus $64 \%$ (95\% CI $58 \%-70 \%)$ for coronary CTA.
The more recent HeartFlow NXT trial further assessed FFR $_{\text {CTA }}$ in 254 patients and generated improved diagnostic accuracy (per-patient accuracy 81\%; 95\% CI: 76\%-85\%). ${ }^{42}$ Improving the diagnostic accuracy of $\mathrm{FFR}_{\mathrm{CTA}}$ in the HeartFlow NXT trial when compared to earlier trials, namely, DISCOVERFLOW and DeFACTO, reflects the substantial refinement in $\mathrm{FFR}_{\mathrm{CTA}}$ technology and physiologic modeling. Subsequently, FFR $_{\text {CTA }}$ (HeartFlow Inc, Redwood City, CA, USA) has gained US Food and Drug Administration approval as a class II Coronary Physiologic Simulation Software Device.

The clinical outcomes and resource utilization of FFR $_{\text {CTA }}{ }^{-}$ guided strategy was recently assessed in the PLATFORM trial. ${ }^{43}$ Five-hundred and eighty-four patients with suspected CAD were enrolled in this trial from eleven European centers and were assigned to receive "usual testing" or FFR ${ }_{\text {CTA }}$. The primary outcome was the percentage of patients with no evidence of obstructive CAD on ICA. Among patients with a planned ICA, no obstructive CAD was found in $12 \%$ of the $\mathrm{FFR}_{\text {СTA }}$ arm and in $73 \%$ of the "usual care" arm (risk difference $61 \%, 95 \%$ CI $53 \%-69 \%, P<0.0001)$. Notably, the $\mathrm{FFR}_{\mathrm{CTA}}$-guided strategy resulted in cancelation of ICA in $61 \%$ of the patients. The authors suggested that the $\mathrm{FFR}_{\mathrm{CTA}}$-guided strategy is a safe and feasible alternative to ICA. ${ }^{43}$

In addition to the above studies, two meta-analyses were conducted to assess the diagnostic performance of $\mathrm{FFR}_{\mathrm{CTA}}{ }^{44,45}$ The first meta-analysis included a total of 609 patients and 1,050 vessels from the three major multicenter trials (HeartFlow NXT Trial, DISCOVER-FLOW study, and DeFACTO study). ${ }^{44}$ Using the merged study results, FFR $_{\text {CTA }}$ demonstrated a powerful diagnostic accuracy when compared to coronary 
CT at per-patient and per-vessel analyses. The second metaanalysis included five studies of high methodological quality; it concluded that FFR $_{\text {CTA }}$ exhibited a good degree of diagnostic accuracy for the detection of ischemia-causing stenosis, when applying FFR as a standard reference. ${ }^{45}$ These findings remained consistent regardless of whether the diagnostic accuracy of FFR $_{\text {CTA }}$ was examined at a per-patient and per-vessel or per-lesion level using the threshold FFR value of $\leq 0.80$.

\section{Clinical effectiveness and economic implications}

FFR $_{\text {CTA }}$ is a novel non-invasive technology that offers both anatomical and functional assessment of major epicardial coronary arteries. As described above, the computation of FFR from coronary CTA is based on fluid dynamics and physiologic models without the need for image acquisition modification, additional radiation, administration of additional contrast or hyperemia-inducing medications including adenosine. Furthermore, the accuracy of the FFR ${ }_{\text {СTA }}$ to identify ischemia-related lesions is superior to traditional functional stress testing as it provides precise anatomical identification of ischemia-related lesions that would otherwise be difficult to identify using other non-invasive modalities.

Similar to invasive FFR-guided management strategy, the use of FFR $_{\mathrm{CTA}}$ has been shown to improve patient outcome and reduce health care costs. ${ }^{46,47} \mathrm{~A}$ simple cost analysis simulation was performed in 96 patients from the DISCOVER-FLOW study to determine the health care cost under various management scenarios. The projected initial management costs were lowest for the coronary CTA-FFR ${ }_{\text {CTA }}$-ICA strategy (US\$7,674) and highest for ICA-visual assessment strategy (US\$10,702).

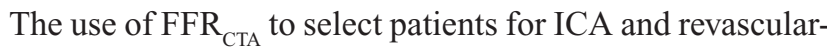
ization would result in $30 \%$ lower costs and $12 \%$ fewer events at 1 year compared to the most commonly used ICA-visual assessment strategy. ${ }^{46}$ Similar results were achieved when a health care cost analysis was conducted on patients from the HeartFlow NXT trial. ${ }^{47}$ It was concluded that the use of the $\mathrm{FFR}_{\mathrm{CTA}}$ strategy to select patients for revascularization would result in 32\% lower costs and 19\% fewer cardiac events in 1 year, compared with the ICA-visual assessment strategy. ${ }^{47}$

\section{Limitations of FFR $\mathbf{R}_{\text {CTA }}$}

$\mathrm{FFR}_{\text {CTA }}$ is emerging as an attractive alternative to invasive FFR. There are, however, several challenges that need to be overcome before FFR $_{\text {CTA }}$ can be translated into routine clinical practice. First, the technique is limited by the same factors that limit the standard coronary CTA namely: calcifications, tachycardia, motion, and misregistration artifacts. These limitations have become particularly important since pre- cise anatomic modeling is an essential step to create an

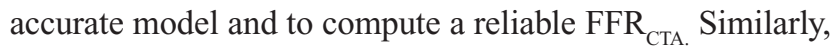
adherence to robust acquisition protocols is important for exact definition of patient-specific boundary conditions. Additionally, the relationships relating myocardial mass to coronary blood flow and resistance to vessel size are prerequisites for generating a patient-specific physiologic model. It is, however, well known that these relationships are highly variable between patients. ${ }^{31}$ Furthermore, FFR $_{\mathrm{CTA}}$ computation assumes a predictable vasodilatation response to adenosine administration, an assumption which is debatable. ${ }^{48}$ Another important limitation is that there are no published data for FFR $_{\text {СTA }}$ in more complex anatomy such as the evaluation of coronary artery bypass grafts or for in-stent restenosis. Finally, the process of $\mathrm{FFR}_{\mathrm{CTA}}$ calculation is time consuming as it takes several hours per exam using specialized software on a high-performance computing platform. An improvement in automation processing time in the future may help in utilizing $\mathrm{FFR}_{\mathrm{CTA}}$ as a desirable assessment tool for day-to-day clinical practice, although the modeling time may not be a significant limitation in clinical practice in the context of stable CAD.

\section{Conclusion}

FFR $_{\text {CTA }}$ is a novel non-invasive technology that offers both anatomical and functional assessment of major epicardial coronary arteries. Similar to an invasive FFR-guided management strategy, the use of FFR $_{\mathrm{CTA}}$ has been shown to improve patients' outcomes and reduce health care costs. FFR ${ }_{\text {CTA }}$ is emerging as an attractive alternative to invasive FFR for low-intermediate risk patients. There are, however, several challenges that need to be overcome before FFR $_{\text {СTA }}$ can be incorporated into routine clinical practice.

\section{Disclosure}

Dr Kakouros has served as an Advisory Board Member to Volcano Corp. The authors have no other conflicts of interest to disclose.

\section{References}

1. GBD 2013 Mortality and Causes of Death Collaborators. Global, regional, and national age-sex specific all-cause and cause-specific mortality for 240 causes of death, 1990-2013: a systematic analysis for the Global Burden of Disease Study 2013. Lancet. 2015;385(9963):117-171.

2. Zipes DP, Camm AJ, Borggrefe M, et al. ACC/AHA/ESC 2006 Guidelines for Management of Patients With Ventricular Arrhythmias and the Prevention of Sudden Cardiac Death: a report of the American College of Cardiology/American Heart Association Task Force and the European Society of Cardiology Committee for Practice Guidelines (writing committee to develop Guidelines for Management of Patients With Ventricular Arrhythmias and the Prevention of Sudden Cardiac Death): developed in collaboration with the European Heart Rhythm Association and the Heart Rhythm Society. Circulation. 2006;114(10):e385-e484. 
3. Gould KL, Lipscomb K, Hamilton GW. Physiologic basis for assessing critical coronary stenosis. Instantaneous flow response and regional distribution during coronary hyperemia as measures of coronary flow reserve. Am J Cardiol. 1974;33(1):87-94.

4. Tonino PA, Fearon WF, De Bruyne B, et al. Angiographic versus functional severity of coronary artery stenoses in the FAME study fractional flow reserve versus angiography in multivessel evaluation. $J \mathrm{Am}$ Coll Cardiol. 2010;55(25):2816-2821.

5. Shaw LJ, Berman DS, Maron DJ, et al. Optimal medical therapy with or without percutaneous coronary intervention to reduce ischemic burden: results from the Clinical Outcomes Utilizing Revascularization and Aggressive Drug Evaluation (COURAGE) trial nuclear substudy. Circulation. 2008;117(10):1283-1291.

6. Pijls NH, De Bruyne B, Peels K, et al. Measurement of fractional flow reserve to assess the functional severity of coronary-artery stenoses. N Engl J Med. 1996;334(26):1703-1708.

7. Pijls NH, van Son JA, Kirkeeide RL, De Bruyne B, Gould KL. Experimental basis of determining maximum coronary, myocardial, and collateral blood flow by pressure measurements for assessing functional stenosis severity before and after percutaneous transluminal coronary angioplasty. Circulation. 1993;87(4):1354-1367.

8. Kakouros N, Rybicki FJ, Mitsouras D, Miller JM. Coronary pressurederived fractional flow reserve in the assessment of coronary artery stenoses. Eur Radiol. 2013;23(4):958-967.

9. De Bruyne B, Baudhuin T, Melin JA, et al. Coronary flow reserve calculated from pressure measurements in humans. Validation with positron emission tomography. Circulation. 1994;89(3):1013-1022.

10. Pijls NH, Van Gelder B, Van der Voort P, et al. Fractional flow reserve. A useful index to evaluate the influence of an epicardial coronary stenosis on myocardial blood flow. Circulation. 1995;92(11):3183-3193.

11. Kern MJ, Samady H. Current concepts of integrated coronary physiology in the catheterization laboratory. $\mathrm{J} \mathrm{Am} \mathrm{Coll} \mathrm{Cardiol.}$ 2010;55(3):173-185.

12. Bech GJ, De Bruyne B, Pijls NH, et al. Fractional flow reserve to determine the appropriateness of angioplasty in moderate coronary stenosis: a randomized trial. Circulation. 2001;103(24):2928-2934.

13. Pijls NH, van Schaardenburgh P, Manoharan G, et al. Percutaneous coronary intervention of functionally nonsignificant stenosis: 5-year follow-up of the DEFER Study. J Am Coll Cardiol. 2007;49(21): 2105-2111.

14. Tonino PA, De Bruyne B, Pijls NH, et al. Fractional flow reserve versus angiography for guiding percutaneous coronary intervention. $N$ Engl $J$ Med. 2009;360(3):213-224.

15. Pijls NH, Fearon WF, Tonino PA, et al. Fractional flow reserve versus angiography for guiding percutaneous coronary intervention in patients with multivessel coronary artery disease: 2-year follow-up of the FAME (Fractional Flow Reserve Versus Angiography for Multivessel Evaluation) study. J Am Coll Cardiol. 2010;56(3):177-184.

16. De Bruyne B, Pijls NH, Kalesan B, et al. Fractional flow reserve-guided $\mathrm{PCI}$ versus medical therapy in stable coronary disease. $N$ Engl $\mathrm{J}$ Med. 2012;367(11):991-1001.

17. Kakouros N, Rade JJ. Role of Fractional-Flow Reserve in Guiding Percutaneous Revascularization in Stable Coronary Artery Disease. Curr Atheroscler Rep. 2015;17(9):52.

18. Levine GN, Bates ER, Blankenship JC, et al. 2011 ACCF/AHA/ SCAI Guideline for Percutaneous Coronary Intervention: a report of the American College of Cardiology Foundation/American Heart Association Task Force on Practice Guidelines and the Society for Cardiovascular Angiography and Interventions. Circulation. 2011;124(23):e574-e651.

19. Authors/Task Force members, Windecker S, Kolh P, et al. 2014 ESC/ EACTS Guidelines on myocardial revascularization: The Task Force on Myocardial Revascularization of the European Society of Cardiology (ESC) and the European Association for Cardio-Thoracic Surgery (EACTS) Developed with the special contribution of the European Association of Percutaneous Cardiovascular Interventions (EAPCI). Eur Heart J. 2014;35(37):2541-2619.
20. Dehmer GJ, Weaver D, Roe MT, et al. A contemporary view of diagnostic cardiac catheterization and percutaneous coronary intervention in the United States: a report from the CathPCI Registry of the National Cardiovascular Data Registry, 2010 through June 2011. J Am Coll Cardiol. 2012;60(20):2017-2031.

21. Lotfi A, Jeremias A, Fearon WF, et al. Expert consensus statement on the use of fractional flow reserve, intravascular ultrasound, and optical coherence tomography: a consensus statement of the Society of Cardiovascular Angiography and Interventions. Catheter Cardiovasc Interv. 2014;83(4):509-518.

22. Schauer DA, Linton OW. National Council on Radiation Protection and Measurements report shows substantial medical exposure increase. Radiology. 2009;253(2):293-296.

23. Budoff MJ, Dowe D, Jollis JG, et al. Diagnostic performance of 64-multidetector row coronary computed tomographic angiography for evaluation of coronary artery stenosis in individuals without known coronary artery disease: results from the prospective multicenter ACCURACY (Assessment by Coronary Computed Tomographic Angiography of Individuals Undergoing Invasive Coronary Angiography) trial. J Am Coll Cardiol. 2008;52(21):1724-1732.

24. Meijboom WB, Meijs MF, Schuijf JD, et al. Diagnostic accuracy of 64-slice computed tomography coronary angiography: a prospective, multicenter, multivendor study. J Am Coll Cardiol. 2008; 52(25):2135-2144.

25. Douglas PS, Hoffmann U, Patel MR, et al. Outcomes of anatomical versus functional testing for coronary artery disease. $N$ Engl J Med. 2015;372(14):1291-1300.

26. SCOT-HEART investigators. CT coronary angiography in patients with suspected angina due to coronary heart disease (SCOTHEART): an open-label, parallel-group, multicentre trial. Lancet. 2015;385(9985):2383-2391.

27. Goldstein JA, Gallagher MJ, O’Neill WW, Ross MA, O’Neil BJ, RaffGL. A randomized controlled trial of multi-slice coronary computed tomography for evaluation of acute chest pain. $\mathrm{J} \mathrm{Am} \mathrm{Coll} \mathrm{Cardiol.}$ 2007;49(8):863-871.

28. Meijboom WB, Van Mieghem CA, van Pelt N, et al. Comprehensive assessment of coronary artery stenoses: computed tomography coronary angiography versus conventional coronary angiography and correlation with fractional flow reserve in patients with stable angina. $J \mathrm{Am}$ Coll Cardiol. 2008;52(8):636-643.

29. Schuijf JD, Wijns W, Jukema JW, et al. Relationship between noninvasive coronary angiography with multi-slice computed tomography and myocardial perfusion imaging. $\mathrm{J} \mathrm{Am} \mathrm{Coll} \mathrm{Cardiol.}$ 2006;48(12):2508-2514.

30. Gaemperli O, Bengel FM, Kaufmann PA. Cardiac hybrid imaging. Eur Heart J. 2011;32(17):2100-2108.

31. Taylor CA, Fonte TA, Min JK. Computational fluid dynamics applied to cardiac computed tomography for noninvasive quantification of fractional flow reserve: scientific basis. $\mathrm{J} \mathrm{Am} \mathrm{Coll} \mathrm{Cardiol.}$ 2013;61(22):2233-2241.

32. Taylor CA, Figueroa CA. Patient-specific modeling of cardiovascular mechanics. Annu Rev Biomed Eng. 2009;11:109-134.

33. Kim HJ, Vignon-Clementel IE, Coogan JS, Figueroa CA, Jansen KE, Taylor CA. Patient-specific modeling of blood flow and pressure in human coronary arteries. Ann Biomed Eng. 2010;38(10):3195-3209.

34. Kim HJ, Jansen KE, Taylor CA. Incorporating autoregulatory mechanisms of the cardiovascular system in three-dimensional finite element models of arterial blood flow. Ann Biomed Eng. 2010;38(7):2314-2330.

35. Glagov S, Weisenberg E, Zarins CK, Stankunavicius R, Kolettis GJ. Compensatory enlargement of human atherosclerotic coronary arteries. N Engl J Med. 1987;316(22):1371-1375.

36. Zarins CK, Zatina MA, Giddens DP, Ku DN, Glagov S. Shear stress regulation of artery lumen diameter in experimental atherogenesis. J Vasc Surg. 1987;5(3):413-420.

37. Kamiya A, Togawa T. Adaptive regulation of wall shear stress to flow change in the canine carotid artery. Am J Physiol. 1980; 239(1):H14-21. 
38. Wilson RF, Wyche K, Christensen BV, Zimmer S, Laxson DD. Effects of adenosine on human coronary arterial circulation. Circulation. 1990;82(5):1595-1606.

39. Taylor CA, Hughes TJ, Zarins CK. Finite element modeling of threedimensional pulsatile flow in the abdominal aorta: relevance to atherosclerosis. Ann Biomed Eng. 1998;26(6):975-987.

40. Koo BK, Erglis A, Doh JH, et al. Diagnosis of ischemia-causing coronary stenoses by noninvasive fractional flow reserve computed from coronary computed tomographic angiograms. Results from the prospective multicenter DISCOVER-FLOW (Diagnosis of Ischemia-Causing Stenoses Obtained Via Noninvasive Fractional Flow Reserve) study. J Am Coll Cardiol. 2011;58(19):1989-1997.

41. Min JK, Leipsic J, Pencina MJ, et al. Diagnostic accuracy of fractional flow reserve from anatomic CT angiography. JAMA. 2012;308(12):1237-1245.

42. Norgaard BL, Leipsic J, Gaur S, et al. Diagnostic performance of noninvasive fractional flow reserve derived from coronary computed tomography angiography in suspected coronary artery disease: the NXT trial (Analysis of Coronary Blood Flow Using CT Angiography: Next Steps). J Am Coll Cardiol. 2014;63(12):1145-1155.

43. Douglas PS, Pontone G, Hlatky MA, et al. Clinical outcomes of fractional flow reserve by computed tomographic angiography-guided diagnostic strategies vs. usual care in patients with suspected coronary artery disease: the prospective longitudinal trial of FFRCT: outcome and resource impacts study. Eur Heart J. 2015;36(47):3359-3367.
44. Li S, Tang X, Peng L, Luo Y, Dong R, Liu J. The diagnostic performance of CT-derived fractional flow reserve for evaluation of myocardial ischaemia confirmed by invasive fractional flow reserve: a metaanalysis. Clin Radiol. 2015;70(5):476-486.

45. Deng SB, Jing XD, Wang J, et al. Diagnostic performance of noninvasive fractional flow reserve derived from coronary computed tomography angiography in coronary artery disease: A systematic review and metaanalysis. Int J Cardiol. 2015;184:703-709.

46. Hlatky MA, Saxena A, Koo BK, Erglis A, Zarins CK, Min JK. Projected costs and consequences of computed tomography-determined fractional flow reserve. Clin Cardiol. 2013;36(12):743-748.

47. Kimura T, Shiomi H, Kuribayashi S, et al. Cost analysis of non-invasive fractional flow reserve derived from coronary computed tomographic angiography in Japan. Cardiovasc Interv Ther. 2015;30(1):38-44.

48. van de Hoef TP, Meuwissen M, Escaned J, et al. Fractional flow reserve as a surrogate for inducible myocardial ischaemia. Nat Rev Cardiol. 2013;10(8):439-452.

\section{Publish your work in this journal}

The Journal of Vascular Diagnostics and Interventions is an international, peer-reviewed journal of diagnostics, focusing on non invasive vascular investigation methods involved in the evaluation of vascular diseases. The journal is committed to the rapid publication in the fields of vascular diseases. Original research, review, case reports, expert

\section{Dovepress}

opinion and commentaries are all considered for publication. The manuscript management system is completely online and includes a very quick and fair peer-review system, which is all easy to use. Visit http://www.dovepress.com/testimonials.php to read real quotes from published authors. 